\title{
Wave beams with orbital angular momentum: a step towards terahertz
}

\author{
B.A. Knyazev ${ }^{1,2}$, V.S. Cherkassky, Yu.Yu. Choporova ${ }^{1,2}$, O.E. Kameshkov ${ }^{1,2}$, G.N. Kulipanov ${ }^{1}$, \\ N.D. Osintseva ${ }^{1,3}$, V.S. Pavelyev ${ }^{4}$, N.A. Vinokurov ${ }^{1,2}$, B.O. Volodkin ${ }^{4}$ \\ ${ }^{1}$ Budker Institute of Nuclear Physics SB RAS, Novosibirsk, Russia, b.a.knyazev@inp.nsk.su \\ ${ }^{2}$ Novosibirsk State University, Novosibirsk, Russia \\ ${ }^{3}$ Novosibirsk State Technical University, Novosibirsk, Russia \\ ${ }^{4}$ Samara National Research University, Samara, Russia
}

\section{Beam formation}

The energy and angular momentum are quantum characteristics of photons. The angular momentum is the sum of the spin and orbital angular momenta. In physical optics, the spin momentum is described as the polarization of light beams, and the orbital angular momentum $(\mathrm{OAM})$ is the rotation of beam along the azimuth. Interest in beams with OAM $[1,2]$, or "vortex beams," appeared shortly after the creation of lasers and increased after the publication of the article [3]. Vortex beams have been formed and investigated in the spectral range from X-ray to radio waves, but only a few publications are devoted to beams with OAM in the terahertz range (see [4] and references therein). In this paper we describe vortex Bessel beams with an average power of $25 \mathrm{~W}$, obtained on the Novosibirsk free-electron laser (NovoFEL) [5].

Vortex beams were formed from the NovoFEL Gaussian beam transformed by binary phase axicons (BPA) with spiral zone structures (Fig. 1, a, b). The zone boundaries are described with the equation $r_{\beta}=p(l \varphi / 2 \pi+\beta / 2)$, where $p=2 \pi / \kappa=3.2 \mathrm{~mm}$ is the period, $\beta=0 \ldots(2 l-1)$, and $\kappa$ is the transverse wave number. The relief depth was calculated for a wavelength $\lambda=141 \mu \mathrm{m}: \quad H=\lambda_{0} / 2(n-1)=29.1 \mu \mathrm{m} \quad(n=3.42)$. Beams with the topological charges $l= \pm 1$ (Fig. 1, c) were formed with the BPA (a), whereas the BPA (b) formed the beams with intertwined helicoids wavefront ( $l= \pm 2$ ) (Fig. 1, d). The average power of the incident Gauss beam was about $100 \mathrm{~W}$. About $50 \%$ of this power was reflected from the silicon axicon, and then $41 \%$ was diffracted into the converging plane wave, which formed the Bessel beam with a power of $25 \mathrm{~W}$. This value can be increased fourfold by the use an antireflection coating and a telescopic system mounted directly behind the axicon.

\section{Beam characteristics}

The radial distribution of the intensities of the obtained beams recorded with a $16.32 \times 12.24 \mathrm{~mm}^{2} \mathrm{mi}-$ crobolometer array [6] moving along the $z$-axis on a translation stage was in good agreement with the Bessel function (Fig. 2), but the Fourier spectrum of transverse spatial-frequency components of the beams measured at the lens focus consisted, instead of rings observed in the case of ordinary Bessel beams, of segments of $2 l$ intertwined arcs (Fig. 2, a, b). Nevertheless, in the rest, the properties of the obtained beams corresponded to the properties of the Bessel beams. In particular, the crosssection of the beams was unchanged over a length of ap- proximately $150 \mathrm{~mm}$ ("non-diffraction beams"). The beams are self-reconstructing after passing through obstacles and randomly inhomogeneous media.

The formation of Bessel beams by means of a binary spiral axicon has an advantage in comparison with the use of the combination of spiral phase plate and axisymmetric axicon [7]. In the latter case, the optical system correctly forms a vortex beam only for wavelengths for which the thickness of the spiral plate grows up as $h(\varphi)=\lambda l \varphi / 2 \pi(n-1)$. On the contrary, a binary phase axicon with spiral zones forms a beam $E(r, \varphi, z)=E_{0} J_{l}(\kappa r) \exp \left[i\left(k_{z} z-l \varphi\right)\right]$ with a given topological charge for any wavelengths, only its diffraction efficiency changing. The diameters of the beam rings are also independent of the wavelength, which can be useful for many applications.

All these experiments showed that the vortex beams obtained by us possess all the properties of Bessel beams for a certain distance.

\section{Beam diagnostics}

The intensity distributions of the beams formed by the axicons are in good agreement with the distributions calculated for the Bessel beams. However, the intensity distributions are identical for both left-handed and righthanded beams and the topological charge cannot be determined from them. The beam helicity parameters were determined in classical diffraction experiments: the interference of vortex and Gaussian beams, rotation of beam obstructed by a half-plane, and the double-slit Young experiment. The Young experiment proved to be the most obvious and simple way to solve this problem.

The diffraction pattern obtained in this experiment for the Bessel beam with the topological charge $l=-2$ is shown in Fig. 3. The fringes are dogleg shaped and can be described with the expression

$$
x(y, z)=-a \tan \left[\frac{m \pi}{l}+\frac{2 \pi a y}{l z \lambda}+\frac{\pi}{2}\right], m=0 \ldots l-1,
$$

where $2 a$ is the distance between narrow slits.

\section{Beam applications}

The advantages of Bessel beams are widely used, and one can assume that they will also find application in the terahertz range. Communication systems and radars are the most obvious uses. Without touching these problems here, we give examples of vortex Bessel beams applied in optical phenomena in which they were not previously investigated.

The first experiment in which the use of vortex beam led to an unusual result was the generation of surface 
plasmon polaritons by the method of beam diffraction at the edge of metal-dielectric interface ("end-fire coupling"). The plasmons were expected to arise at each intersection of the Bessel beam rings with the surface boundary. However, depending on the direction of the beam twist, plasmons were generated only on one side of the optical axis. This effect, which has not been clearly explained yet, can be used as a new type of binary plasmonic switch.

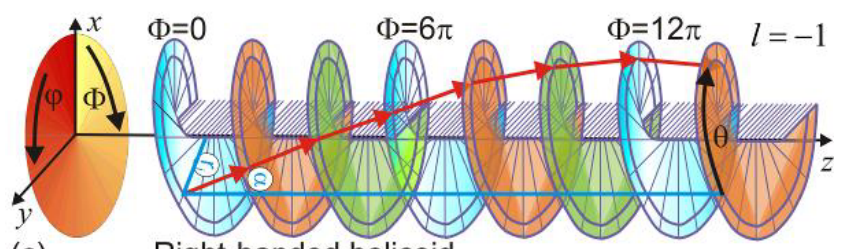

(c) Right-handed helicoid

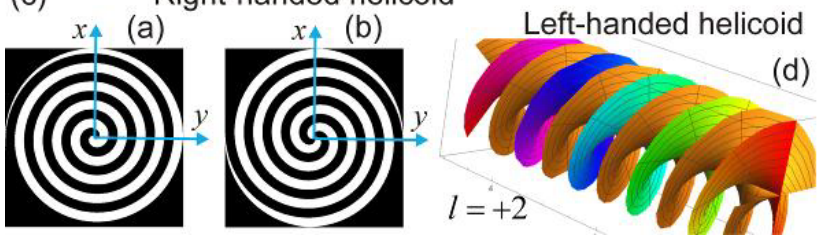

Fig. 1. Binary phase axicons with spiral zones (phase shift by $\pi$ at $\lambda=141 \mu \mathrm{m}$ ) for forming beams with topological charges $l=-1(a)$ and $l=+2(b) ;(c, d)$ helicoidal wavefronts

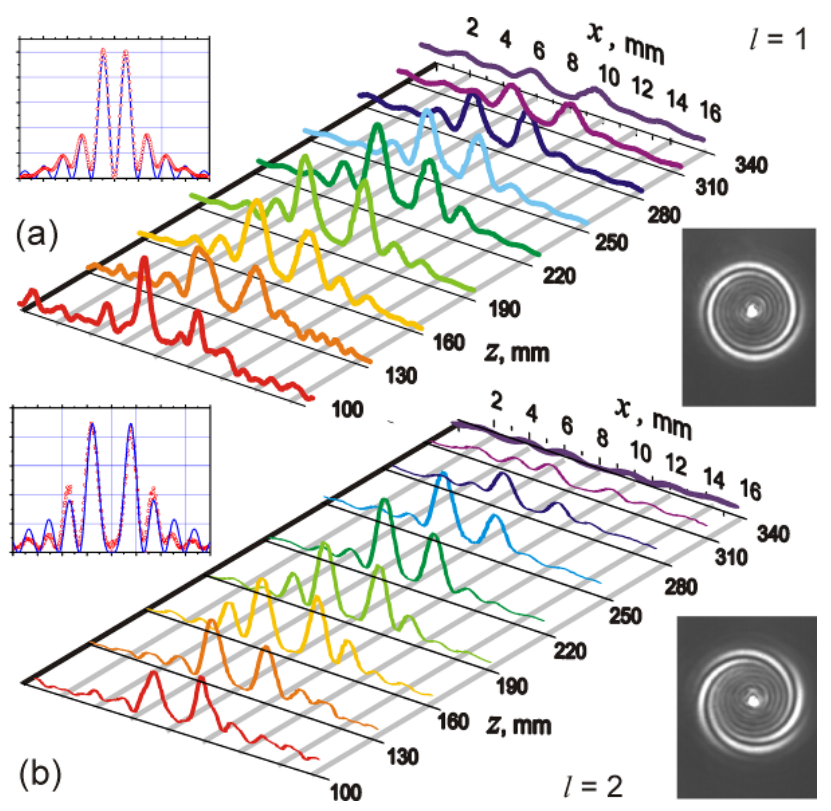

Fig. 2. Cross-sections of beams with topological charges $l=-1(a)$ and $l=+2(b)$ vs. distance. Left insets: comparison of the Bessel functions with beam cross-sections. Right insets: image of beams in lens focal plane (spatial frequency distribution)

We also investigated for the first time the passage of vortex beams through amplitude and phase periodic gratings, that is, an analog of the Talbot effect. Experiments and numerical calculations have shown that, as in the classical Talbot effect, periodic diffraction patterns are formed in the main and fractional Talbot planes, depending however on a larger number of parameters than the classical effect. The transformation of a large vortex beam into a periodic lattice of small vortex beams was demonstrated.
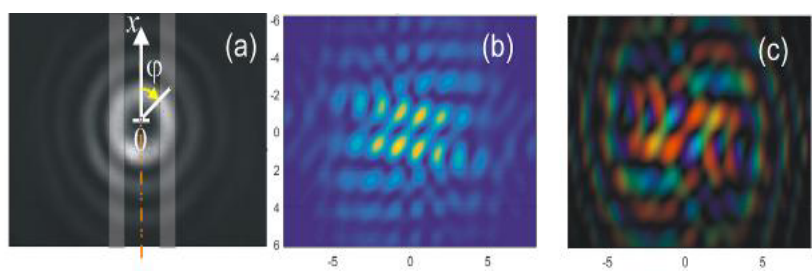

Fig. 3. (a) Double slit with spacing of $4.5 \mathrm{~mm}$, illuminated by vortex beam; $(b)$ The experimentally observed diffraction pattern $40 \mathrm{~mm}$ behind the slits reveals that the beam topological charge is equal to $l=-2 ;(c)$ (color online) Simulated diffraction pattern in which colors show phase distribution from 0 (blue) to $\pi$ (red)

\section{Acknowledgements}

Experiments were supported by RFBR grant 15-02-06444. The axicons were fabricated under supported by the Ministry of Education and Science of the Russian Federation (project 16.7894.2017/6.7). The terahertz beamline was designed under support of RSF grant 14-50-00080. The experiments engaged equipment belonging to the Siberian Synchrotron and Terahertz Radiation Center.

\section{References}

1. Baranova, N.B., Zeldovich, B.Ya. Dislocations of the wave-front surface and zeros of the amplitude // Sov. Phys. JETP 1981. V. 53, P. 925-929.

2. Bazhenov, V.Yu., Vasnetsov, M.V., Soskin, M.S. Laser beams with screw dislocations in their wavefronts // JETP Lett. 1990. V. 52, P. 429-431.

3. Allen, L., Beijersbergen, M.W., Spreeuw, R.J.C., Woerdman, J.P. Orbital angular momentum of light and the transformation of Laguerre-Gaussian laser modes // Phys. Rev. A. 1992. V. 45, $8185-8189$.

4. Knyazev, B.A., Choporova, Yu.Yu., Mitkov, M.S., Pavelyev, V.S., Volodkin, B.O. Generation of terahertz surface plasmon polaritons using nondiffractive Bessel beams with orbital angular momentum // Phys. Rev. Lett. 2015. V. 115, 163901 (5 p.).

5. Kulipanov, G.N., et al. Novosibirsk Free Electron Laser-Facility Description and Recent Experiments // IEEE Trans. Terahertz Sci. Technol. 2015. V. 5. P. 798-809.

6. Dem'yanenko, M.A., Esaev, D.G., Marchishin, I.V., Ovsyuk, V.N., Fomin, B.I., Knyazev, B.A., Gerasimov V.V., Application of uncooled microbolometer detector arrays for recording radiation of the terahertz spectral range // 2011. Optoelectron., Instrum. Data Process. V. 47, P. 508-512.

7. Wei, X., Liu, C., Niu, L., Zhang, Z., Wang, K., Yang, Z., Liu, J. Generation of arbitrary order Bessel beams via 3D printed axicons at the terahertz frequency range // Appl. Opt. 2015. V. 54. P. 10641-10649. 